\begin{tabular}{ll|l}
\cline { 2 - 3 } & \multicolumn{2}{l}{ Intervent Neurol 2016;5:29-38 } \\
\cline { 2 - 3 } & $\begin{array}{l}\text { DOI: 10.1159/000444306 2016 S. Karger AG, Basel } \\
\text { Published online: March 4, 2016 }\end{array}$ & $\begin{array}{l}\text { 1664-9737/16/0052-0029\$39.50/0 } \\
\text { www.karger.com/ine }\end{array}$ \\
\hline
\end{tabular}

\title{
Advances in the Pathogenesis, Diagnosis and Treatment of Bow Hunter's Syndrome: A Comprehensive Review of the Literature
}

\author{
Guangxin Duan ${ }^{a}$ Jiaping $\mathrm{Xu}^{\mathrm{a}}$ Jijun Shi ${ }^{\mathrm{a}}$ Yongjun $\mathrm{Cao}{ }^{\mathrm{a}, \mathrm{b}}$ \\ a Department of Neurology, The Second Affiliated Hospital of Soochow University, and \\ bjiangsu Key Laboratory of Translational Research and Therapy for Neuro-Psycho-Diseases, \\ Institute of Neuroscience, Soochow University, Suzhou, China
}

\section{Key Words}

Bow hunter's syndrome · Vertebral artery · Vertebrobasilar insufficiency · Endovascular therapy

\begin{abstract}
Bow hunter's syndrome (BHS), also known as rotational vertebral artery (VA) occlusion syndrome, is a rare yet treatable type of symptomatic vertebrobasilar insufficiency resulting from mechanical occlusion or stenosis of the VA during head and neck rotation or extension. The symptoms of BHS range from transient vertigo to posterior circulation stroke. The underlying pathology is dynamic stenosis or compression of the VA by abnormal bony structures with neck rotation or extension in many cases, such as osteophyte, disc herniation, cervical spondylosis, tendinous bands or tumors. Imaging approaches, such as Doppler sonography, computed tomography and angiography, as well as magnetic resonance imaging and angiography, are widely used in the diagnosis and evaluation of this syndrome. Digital subtraction angiography with head rotation remains the gold standard diagnostic method. Conservative management, surgery and endovascular procedures are the three major treatment methods for BHS, whereas some symptomatic patients may need operative treatment including surgery and endovascular procedures when conservative management is not adequate.
\end{abstract}

(c) 2016 S. Karger AG, Basel

\section{Introduction}

Bow hunter's syndrome (BHS), also known as rotational vertebral artery (VA) syndrome, refers to symptomatic vertebrobasilar insufficiency (VBI) caused by mechanical occlusion or compression of the VA at the atlantoaxial or subaxial level during neck and head rotation. It was first reported by Sorensen [1] in 1978 in a patient who developed Wallenberg syndrome 

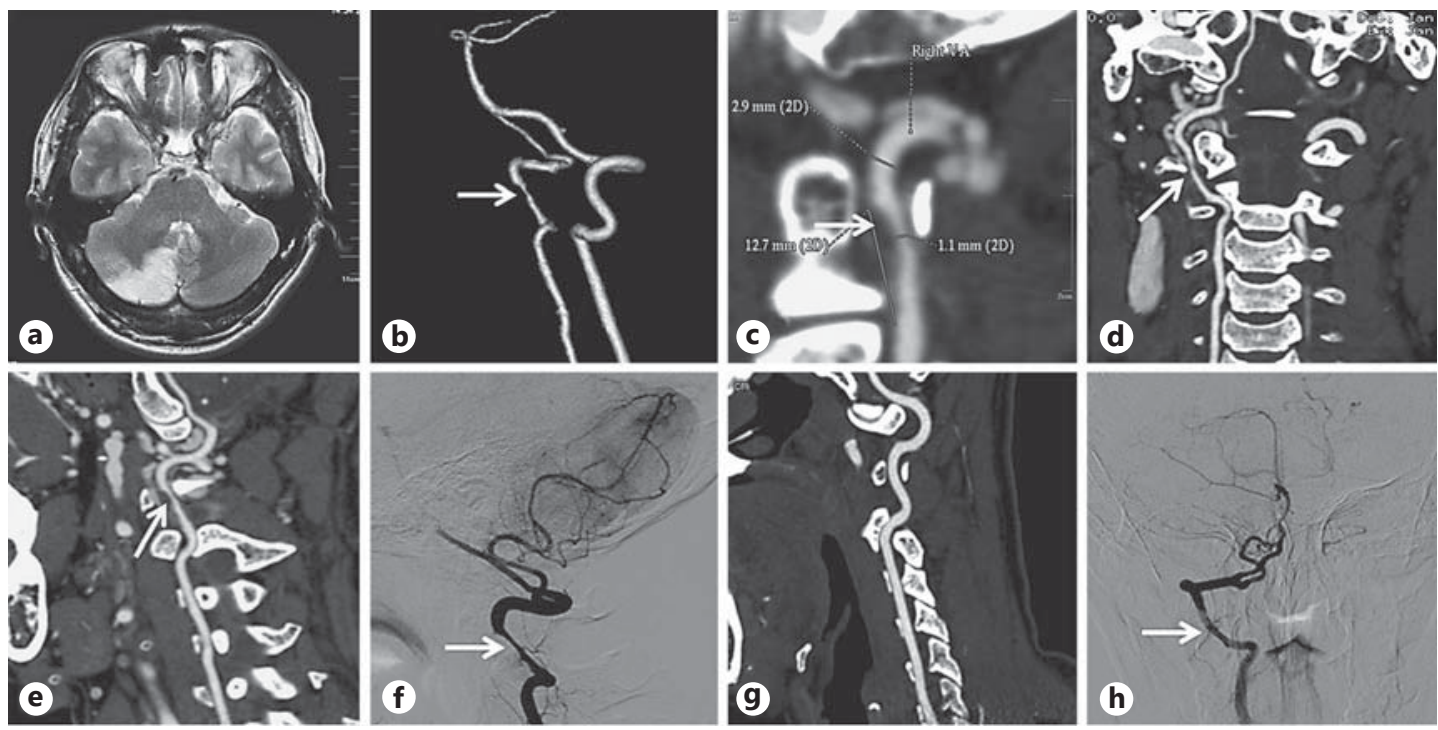

Fig. 1. Neurological images of a case diagnosed with BHS. This is a 37-year-old male patient who was admitted complaining of dizziness with nausea and vomiting for 5 days. He was a porter in a logistic company without cerebrovascular risk factors. a MRI (T2-weighted) revealed a new infarction lesion in the right cerebellar hemisphere. b-f Severe stenosis in the V3 segment (C1-C2) of the right VA (arrow). g, h The left VA is normal by CTA and DSA.

and additional pyramidal tract affection secondary to a posterior circulation infarction during archery practice; accordingly, the term 'bow hunter's syndrome' was introduced. Compared with vertebral artery cervical spondylopathy, which is caused by cervical vertebral bodies or their accessories, and usually presents with neck pain, BHS has more complicated causes and seldom presents with pain, although they both have the same symptoms as VBI.

Due to the limited number of case reports and case series, the exact incidence of BHS is still unknown. Jost and Dailey [2] summarized 126 cases identified in a literature review, while Rastogi et al. [3] analyzed the information of 153 patients with BHS given in the literature. Moreover, due to the rarity of the pathology, there are still no guidelines on the diagnosis and management of BHS. Conservative management, surgery and endovascular therapy are the three main treatment methods for this relatively rare syndrome.

In this review, we present the epidemiology, etiology and pathogenesis of BHS. The clinical manifestations, diagnosis, evaluation and treatment methods will also be discussed, with references to the relevant literature and data.

\section{Epidemiology and Characteristics}

BHS may occur in any period of life but typically presents in the 5th-7th decade, affecting more male than female patients. Zaidi et al. [4] analyzed 14 patients diagnosed with BHS referred to the Barrow Neurological Institute, with a mean age of 58.3 years. The percentage of males was 54.5\%. Recently, Jost and Dailey [2] analyzed 126 patients diagnosed with BHS, including 2 pediatric patients, 3 adolescents and 121 adults, with a mean age of $57 \pm 11$ years of all the adults at diagnosis and with a male-to-female distribution of about 2:1 overall. Rastogi et al. [3] reviewed 153 patients with BHS given in the literature, with a mean age of 53 years, after having excluded 10 pediatric patients ( $<18$ years), and 
there were 100 males and 45 females. In addition to old age, the patients in this group also had some comorbidities or cerebrovascular risk factors, such as hypertension, hyperlipidemia, osteoarthritis, diabetes, smoking or coronary artery disease. Most patients suffered from two or more of these incidents [3-5]. Due to the relatively small sample, further research should be conducted to acquire additional information about the epidemiology of BHS in the future.

\section{Etiology and Pathogenesis}

Various etiologies have been illustrated in the literature for VA compression or stenosis of this syndrome. Anatomically, the VA originates from the subclavian artery and courses through six foramina transversaria of the cervical vertebrae, passing through the groove on the surface of the arch of the atlas and then penetrating the dura mater [6]. Because of this anatomical course, the vertebral arteries are often affected by head motion. Here, we introduce some of the etiologies and categories of BHS.

Firstly, primary BHS should be distinguished from acquired BHS. The former is often caused by disc herniation [2, 4], abnormal bony structures (e.g. osteophyte [7-9], idiopathic skeletal hyperostosis [10] and spondylotic changes [11]), ligament [4], neck muscle hypertrophy [12], VA dissection [13] and some other causes like an instability of the uncovertebral joint [14]. Osteophytes, or bone spurs, are the most common etiology of BHS [3]. Acquired BHS is part of the complications of cervical spondylosis and surgery [10], like the VA sacrificed during the treatment of an aneurysm [15], neck or head injury [16-19] or sports incidents [20] and so on.

BHS can also be categorized into pediatric BHS, juvenile BHS and adult BHS, based on patient age at final diagnosis with BHS. This has not been mentioned in the literature so far. Pediatric patients may present with congenital anomalies such as bony malformation at the atlantoaxial level or a suboccipital bony protuberance impinging on the VA [21, 22], while juvenile BHS patients may present with an occipital condylar bone spur [20], an anomalous bypass of the VA [23] or anomaly of the cervical spine [24]. The etiologies of adult BHS are similar to those of primary BHS mentioned above. Most of the patients with BHS are adults, and the compressive causes are related to the exact location. Therefore, Cornelius et al. [21] recommended dividing BHS into atlantoaxial BHS, subaxial BHS and mixed type.

In the class of atlantoaxial BHS, just as its name implies, the lesion is exactly located at the atlantoaxial level because of an ossified or thickened atlanto-occipital membrane [19,25], a dural fold in the foramen magnum [26], an assimilated posterior ring of the atlas [27], an accessory ossicle behind the atlanto-odontoid junction [17], erosive rheumatoid arthritis of C1-C2 [28], C1-C2 facet hypertrophy [29] or others. In subaxial BHS, the ipsilateral VA was often compressed at the subaxial level by a bony spur of a hypertrophied uncovertebral joint [30] or by a laterally herniated disc [31]. Finally, a mixed type may be distinguished, which may involve bilateral VA stenosis - one at C1-C2 and the other at the subaxial level - as indicated in a case reported by Kimura et al. [32].

Recently, Jost and Dailey [2] have summarized the information of 126 patients with BHS and pointed out that the occlusion was mostly located between C3 and C7 (58\%), followed by C1-C2 (36\%), and with just 6\% proximal to C7 and distal to C1. Also, nondominant VAs were occluded, which resulted in symptoms common of patients with BHS $[9,33]$. There was almost no case report where patients presented with BHS without hemodynamic changes except for 1 case reported by Saito et al. [23] who had an anomalous bypass of the VA. However, from the analysis of Rastogi et al. [3], we can see that the level of the atlantoaxial (C1-C2) joint is most commonly involved $(99 / 142)$, which was significantly $(p<0.0001)$ higher than any other level of VA occlusion. 
The underlying pathogenesis is hemodynamic and/or thromboembolic because of endothelial damage by repetitive shear stress [21]. Alternatively, thrombus formation may be attributed to blood flow stasis in the VA, a well-accepted mechanism underlying hemodynamic BHS. In most BHS, the dominant VA is compressed at the atlantoaxial or subaxial level, and the opposite VA is often hypoplastic, stenotic or occlusive by arteriosclerotic changes. Overall, the left VA is involved in a significantly $(\mathrm{p}<0.0001)$ higher number of BHS patients than the right VA or even bilateral VA involvement, which might be attributed to the fact that the left VA is dominant in $50 \%$ of people, whereas right dominance is found in only $25 \%$ [3, 34]. Husni and Storer [35] reported 23 patients with VBI induced by the rotational occlusion of one VA. The opposite VA was hypoplastic or absent in 22 patients and narrowed at its origin in 1 patient. Meanwhile, posterior communicating arteries are generally hypoplastic or aplastic, and the anterior cerebral circulation is incapable of providing enough collateral blood flow to the posterior cerebral circulation. The aplastic VAs often terminate in the posterior inferior cerebellar artery (PICA) and may be the culprit artery resulting in bow hunter's stroke [36]. It was reported by Darkhabani et al. [5] that 3 of 4 patients eventually treated with stenting exhibited a dominant left VA with a hypoplastic or stenotic contralateral VA; in the other patient, VBI was due to stenosis of the nondominant VA, which terminated in the PICA and resulted in vertiginous episodes with head movement related to ipsilateral PICA ischemia. Mechanical occlusion of bilateral patent VAs has also been reported in some cases $[11,37]$. All of these situations may lead to hemodynamic changes in the posterior circulation system and contribute to the symptoms of $\mathrm{VBI}$, even resulting in posterior circulation infarction under some circumstances.

In other cases, thrombus formation or artery-to-artery embolism may account for the occurrence of BHS $[20,23]$ that had already developed into an ischemic stroke, which may be secondary to VA dissection because of bony spur [20] or some other cause. As the exact mechanism underlining BHS remains unclear, artery-to-artery embolism due to intimal and vascular damage caused by repeated VA compression may be one of the mechanisms $[23,38]$. Repeated intimal injury and vascular damage promote platelet aggregation and activate coagulation mediators, and consequently, thrombus formation occurred [39]. This is supported by some case reports in which patients with repetitive vertebrobasilar infarction and VA occlusion on head rotation had a mobile thrombus at the compression site $[24,38]$.

\section{Clinical Manifestations}

The symptoms of BHS vary from transient ischemic attacks to potentially devastating posterior circulation strokes, which can be repeatedly evoked by rotation or extension of the head and neck. The symptoms usually resolve immediately as the head returns to a neutral position. The threshold angles that evoke the symptoms are $30^{\circ}[25], 40^{\circ}[40], 45^{\circ}[15,29,33$, 41-43], $60^{\circ}[13,19,42,44], 80^{\circ}$ [45] and $90^{\circ}$ [46]. Velat et al. [30] summarized in their paper that classic presentations of this syndrome included dizziness, vertigo, nystagmus, nausea with associated emesis, Horner's syndrome, syncope, and motor or sensory deficits that occur with head rotation (relatively rare in BHS). Cornelius et al. [21] reported 5 patients with BHS; their main symptoms were vertigo and visual blur during head rotation, extreme rotation which regularly induced syncope, and 1 patient even developed a posterior circulation infarction. Zaidi et al. [4] analyzed 14 patients referred to the Barrow Neurological Institute during 1999-2013 with signs and symptoms that were possibly indicative of BHS. The primary symptoms were syncope/presyncope $(54.5 \%)$, vertigo (36.7\%), double vision $(18.2 \%)$, headache (9.1\%) and paresthesias (9.1\%). Recently, Jost and Dailey [2] summarized the relevant data from 126 patients diagnosed with BHS and pointed out that the most commonly 
reported symptoms were syncope, near-syncope, drop attacks, vertigo, intermittent dizziness and impaired vision. Some other symptoms, including positional tinnitus, nausea, headache, presyncopal sensation, near loss of consciousness, hemiparesis and numbness were also reported. Rastogi et al. [3] observed that vertigo and syncope were present in 28 and $26 \%$ of the cases, respectively, and these were the most prevalent symptoms noticed in patients with BHS in their review. However, in our opinion, all these presentations are not specific to BHS, and they may also appear in vestibular disorders, carotid stenosis, cardiac disease and some other situations. The diagnosis of BHS should not only be based on clinical and angiographic findings, but also on neuroanatomical and hemodynamic effects of head rotation [47].

\section{Diagnosis and Evaluation}

Concerning the means of evaluation and diagnosis of BHS, computed tomography and angiography (CT/CTA), magnetic resonance image and angiography (MRI/MRA) as well as neurosonography, which can detect abnormal bony structures, infarction lesions or stenotic arteries, are routine examinations. The final diagnosis should be made upon digital subtraction angiography (DSA), showing the patent arteries in neutral position and the stenotic arteries in the rotated position (fig. 1).

In the early reports, plain X-rays were used to demonstrate cervical spondylosis, abnormality, instability and narrowing or severe bony proliferative changes $[25,29,33,41]$, which may suggest a diagnosis of BHS.

Nowadays, CTA and MRA with or without 3D reconstruction are often performed for the diagnosis in patients suspected of having BHS. Angiographies in neutral and rotated positions should be acquired. Since the symptoms were evoked just a few seconds after the rotation of the head or neck, CTA and MRA (at least 2 min required) were not as quick and accurate as DSA. Both these procedures have potential risks of causing severe symptoms, even posterior circulation infarction, when the patients rotate their heads to the symptomatic side. CTA with reconstructions can give very precise information about compressing elements and the topographical relationships between the vascular anatomy and the surrounding structures in the same image [48]. MRI has the advantage of documenting any ischemic event. In addition, intraoperative dynamic angiography is useful to determine the hemodynamic resolution of BHS and may therefore potentially minimize the amount of decompression [30]. Meanwhile, rotational vertigo may occur due to either vestibular or vascular abnormalities. The balloon occlusion test of the VA during angiography is also valuable in the diagnosis of so-called BHS and in the guidance of its surgical management [6].

Neurosonography, including extracranial and transcranial Doppler (TCD) sonography, as well as duplex ultrasonography $[17,18,21,42,45]$ are also widely used prior to, during and immediately after treatments to confirm the lesions or therapeutic effects.

Recently, TCD ultrasonography has been increasingly employed as a noninvasive, inexpensive, safe and portable technique for measuring cerebrovascular function. It permits continuous and bilateral recording of cerebral blood flow velocity through the major intracranial vessels. Reproducible hemodynamic changes can be easily found by TCD in those patients diagnosed with BHS. When the patient slowly turned his head $80^{\circ}$ to the left, flow velocities gradually decreased and finally became absent in the posterior cerebral artery, basilar artery and right VA. The patient felt faint immediately after blood flow ceased in these vessels during TCD. Blood flow velocity quickly increased above normal levels and symptoms disappeared when head rotation was released into the neutral position [45]. Intracranial vessel stenosis, vasospasm, spontaneous emboli and collateral circulation can also be detected by TCD. Therefore, TCD ultrasonography can be a significant alternative procedure to screen 
Duan et al.: Advances in the Pathogenesis, Diagnosis and Treatment of Bow Hunter's

for those patients with signs and symptoms suspicious of this syndrome. Moreover, intraoperative Doppler ultrasonography can be applied directly upon the VA to provide an unrestricted real-time assessment of the surgical decompression for BHS [17].

Cerebral blood flow scintigraphy and single-photon emission CT scanning have also been used to document the blood flow abnormalities in patients with BHS [31]. A decrease in cerebral blood flow may be found by scintigraphy in the lower part of the left cerebellar hemisphere with head rotation.

Electrophysiological examinations, including somatosensory evoked potentials, motor evoked potentials and brain stem auditory evoked response, also served as baseline information before and after some surgical management [15, 17, 21, 43].

Except for these examinations mentioned above, dynamic DSA has been widely approved as the gold standard with the advantages of accuracy, quickness and less trauma [21]. Dynamic angiography is a valuable tool in the diagnosis, localization and treatment planning for BHS. It identifies the VA that is under mechanical compression with head rotation along with the level of compression in most cases. The patent VA or VAs in the neutral position will be found stenotic or occlusive in the rotated position in those typical cases. In addition, angiography is useful in the evaluation of the status of the contralateral VA with respect to hypoplasia or stenosis and the degree of collateral flow from the anterior circulation through the posterior communicating artery. Patients with reproducible symptoms of VBI after head rotation should be evaluated with many examinations from TCD to CTA or MRA combined with CT or MRI of the cervical spine or brain. Finally, dynamic DSA with head rotation should be performed to make a definite diagnosis of BHS, to locate the VA compression site and to optimize treatment planning for BHS [49].

\section{Treatment Methods}

Today, there are still no standard treatment options for BHS because of its rarity. Multiple management strategies have been presented in the literature including conservative, surgical and, more recently, endovascular intervention [21].

Some patients may receive conservative therapies only [1, 25, 43, 47], including avoiding head and neck rotation using a neck brace or collar, antiplatelet or anticoagulation therapy (aspirin, clopidogrel, cilostazol or warfarin).

Choi etal. [50] analyzed the outcome of 21 patients diagnosed with rotational VA occlusion (RVAO) with a median follow-up period of 37.5 months, and most of them received conservative treatment, except for 2 cases who received C1-C2 fusion. None of the 19 patients under conservative treatment developed posterior circulation stroke, and 4 of them showed resolution of the symptoms during the follow-up, possibly because of spontaneous resolution of the extrinsic compression or central adaptation. This suggests that conservative treatments might be safe and might be considered as a first-line treatment in RVAO [50]. However, a different conclusion has been drawn in another analysis, which revealed that surgery was associated with a higher favorable outcome, where only $37 \%$ of the patients who received a conservative approach had favorable outcomes $(p<0.0001)$ [3].

Other patients may have received conservative treatment at the beginning, and then, had to change to surgical treatments like decompression or fusion when the symptoms had not improved $[14,51]$. Also, there was a case who applied for coil embolization of his right VA because of a focal dissection after a 3-month conservative therapy with no improvement of his symptoms [20]. Another patient refused any treatment because his symptoms only occurred with extreme lateral head rotation, and thus, he just received some suggestions to avoid situations which may evoke or aggravate his symptoms [12]. 
However, those patients who were treated conservatively with medication may require surgery because of repeated, aggravated symptoms.

In the case of surgical treatment methods for BHS, the pathogenesis of the hemodynamic abnormality must be considered in individual cases, and the most effective and least invasive procedure should be selected. Surgical treatments, often involving surgical decompression of the VA or C1-C2 fusion, were performed in the majority of cases resulting from abnormal bony structures, disc herniation, instability of the joint and others. In prior reports, many patients underwent decompression through an anterior or posterior approach according to the site of compression [15, 17, 19, 21, 30, 33, 40, 41, 51-53]. In another 2 cases, 1 patient finally received anterior intervertebral fusion because of uncovertebral joint instability [14], and the other posterior fixation and fusion because of facet hypertrophy [29]. Both of these two methods can immediately relieve the symptoms. Whether choosing decompression or fusion, the advantages and disadvantages as well as etiologies should be taken into consideration.

Patients who received decompression had no restrictions of their range of head movements, which may be preferable to those who were drivers, but they had potential risks of restenosis that may result in symptom recurrence, even posterior circulation infarction. In 9 patients diagnosed with BHS and treated with posterior decompression of VA reported by Matsuyama et al. [47], reocclusion of the operated VA was recognized in 3 patients $(3 / 9,33 \%)$ after surgery, and 2 of them had more severe neurologic symptoms than preoperatively. The causes of postoperative reocclusion are as follows: bony structures such as the transverse process or the groove of the atlas, which protect the VA from adhesion or compression of the paravertebral muscle or connective tissues, are surgically removed. This manipulation promotes adhesion between the VA and soft tissues around it, and then, the VA will become more compressed, restricted and easily obstructed by head rotation [47].

A completely different concept from vascular decompression is that of performing C1-C2 fusion. This procedure involves using a bone graft from the iliac crest and wedging it between the posterior arch of the atlas and the lamina of the axis. Surgical intervention through C1-C2 fusion has been used to permanently restrict neck movements and thereby prevent VA compression. It has the serious disadvantage that it markedly reduces the range of the head rotation, but it has a lower risk of restenosis, and life-threatening neurological sequelae can be eliminated with certainty. In the study of Matsuyama et al. [47], it was revealed that all patients felt more comfortable than preoperatively even though their head movements were moderately or severely restricted. These results were all mentioned in the prior paper by Matsuyama et al. [47], which, however, included only 17 patients. When the VAs of patients are compressed bilaterally or ipsilaterally and there is an unstable joint at the same time, the combination of decompression and fusion should be adopted [11, 21, 42, 46, 54]. A rare case has been reported by Kan et al. [16] who developed disabling symptoms of VBI after trauma when the patient rotated his head to the right. Dynamic angiography demonstrated a chronic dissection and stasis of flow in the right VA when the head was rotated to the right; with no obvious site of focal compression, the patient ultimately underwent a PICA-to-PICA in situ bypass to revascularize his right PICA territory with complete symptom resolution. Fusion surgery, instead of bilateral decompression, may also be suitable for those with bilateral RVAO with the difficulty to identify the proper affected side [55].

In order to minimize the surgical risks, surgery should be performed only in centers with high competence in vascular and complex spinal procedures.

In those cases with contralateral VA occlusion or hypoplasia, a direct surgical approach may be a reasonable treatment. However, in the setting of contralateral VA stenosis, angioplasty with or without stent placement of the stenotic portion will provide a sufficient effect [44]. Moreover, this endovascular procedure is less invasive than direct surgical approaches 
[44]. The principle mechanism of these self-expanding stents is to increase the resistance of the VA against compression and to improve its ability to return to its original shape after compression, especially in the V2 segment.

In a case series reported by Darkhabani et al. [5], treatment with VA stenting was performed on the dominant VAs in 3 of 4 patients and in the nondominant VA in 1 patient, in whom the symptoms were attributed to ipsilateral PICA ischemia.

Endovascular approaches described in the prior reports included coil embolization [20, $24]$ and angioplasty with stenting [5, 44]. Treatment decisions should be made upon the specific situation of each patient, like VA dissection, severe stenosis at the origin of the VA or others. The advantages of VA stent placement compared with cervical vertebral fusion include the minimally invasive nature of endovascular therapy and the ability to preserve the neck range of motion.

As the long-term data of endovascular treatment for BHS mainly came from case reports and small clinical series, large randomized clinical trials are needed to evaluate the effectiveness of angioplasty or stenting for BHS.

\section{Conclusion}

Adult patients, especially males and those aged 50-70 years, presenting with symptoms in accordance with VBI after they rotated their head and neck more than $30^{\circ}$ should be suspected of having BHS. Routine examinations, including CT/CTA, MRI/MRA and neurosonography, can demonstrate abnormal surrounding structures, infarction lesions and stenotic arteries. The final diagnosis should be made upon DSA, showing the patent arteries in the neutral position and the stenotic arteries in the rotated position. All relevant factors including the severity, etiology, occupation, lifestyle and some other factors should be taken into consideration in choosing the treatment method. We recommend adopting the surgical treatment or endovascular therapy to eradicate the causative factors.

\section{Acknowledgment}

This paper was financially supported by grants from the National Natural Science Foundation of China (No. 81471195)

\section{Disclosure Statement}

The authors have no conflicts of interest to disclose.

\section{References}

1 Sorensen BF: Bow hunter's stroke. Neurosurgery 1978;2:259-261.

2 Jost GF, Dailey AT: Bow hunter's syndrome revisited: 2 new cases and literature review of 124 cases. Neurosurg Focus 2015;38:E7.

3 Rastogi V, Rawls A, Moore 0, et al: Rare etiology of bow hunter's syndrome and systematic review of literature. J Vasc Interv Neurol 2015;8:7-16.

4 Zaidi HA, Albuquerque FC, Chowdhry SA, Zabramski JM, Ducruet AF, Spetzler RF: Diagnosis and management of bow hunter's syndrome: 15-year experience at barrow neurological institute. World Neurosurg 2014;82: 733-738.

5 Darkhabani MZ, Thompson MC, Lazzaro MA, Taqi MA, Zaidat 00: Vertebral artery stenting for the treatment of bow hunter's syndrome: report of 4 cases. J Stroke Cerebrovasc Dis 2012;21:908.e1-e5. 
6 Hanakita J, Miyake H, Nagayasu S, Nishi S, Suzuki T: Angiographic examination and surgical treatment of bow hunter's stroke. Neurosurgery 1988;23:228-232.

7 Ding D, Mehta GU, Medel R, Liu KC: Utility of intraoperative angiography during subaxial foramen transversarium decompression for bow hunter's syndrome. Interv Neuroradiol 2013;19:240-244.

8 Anaizi AN, Sayah A, Berkowitz F, McGrail K: Bow hunter's syndrome: the use of dynamic magnetic resonance angiography and intraoperative fluorescent angiography. J Neurosurg Spine 2014;20:71-74.

9 Thomas B, Barreau X, Pointillart V, Sibon I, Renou P: Endovascular embolization of a nondominant vertebral artery compressed by an osteophyte to prevent recurrence of vertebrobasilar infarctions. J Stroke Cerebrovasc Dis 2015;24:e257-e259.

10 Healy AT, Lee BS, Walsh K, Bain MD, Krishnaney AA: Bow hunter's syndrome secondary to bilateral dynamic vertebral artery compression. J Clin Neurosci 2015;22:209-212.

11 Fleming JB, Vora TK, Harrigan MR: Rare case of bilateral vertebral artery stenosis caused by C4-5 spondylotic changes manifesting with bilateral bow hunter's syndrome. World Neurosurg 2013;79:799.E1-E5.

12 Sarkar J, Wolfe SQ, Ching BH, Kellicut DC: Bow hunter's syndrome causing vertebrobasilar insufficiency in a young man with neck muscle hypertrophy. Ann Vasc Surg 2014;28:1032.e1-e10.

13 Yamaguchi Y, Nagasawa H, Yamakawa T, Kato T: Bow hunter's syndrome after contralateral vertebral artery dissection. J Stroke Cerebrovasc Dis 2012;21:916.e7-e9.

14 Yoshimura K, Iwatsuki K, Ishihara M, Onishi Y, Umegaki M, Yoshimine T: Bow hunter's stroke due to instability at the uncovertebral C3/4 joint. Eur Spine J 2011;20(suppl 2):S266-S270.

15 Ikeda DS, Villelli N, Shaw A, Powers C: Bow hunter's syndrome unmasked after contralateral vertebral artery sacrifice for aneurysmal subarachnoid hemorrhage. J Clin Neurosci 2014;21:1044-1046.

16 Kan P, Yashar P, Langer DJ, Siddiqui AH, Levy EI: Posterior inferior cerebellar artery to posterior inferior cerebellar artery in situ bypass for the treatment of bow hunter's-type dynamic ischemia in holovertebral dissection. World Neurosurg 2012;78:553.e15-e17.

17 Whitmore RG, Simon SL, Hurst RW, Nisenbaum HL, Kasner SE, Zager EL: Bow hunter's syndrome caused by accessory cervical ossification: posterolateral decompression and the use of intraoperative Doppler ultrasonography. Surg Neurol 2007;67:169-171.

18 Duval EL, Van Coster R, Verstraeten K: Acute traumatic stroke: a case of bow hunter's stroke in a child. Eur J Emerg Med 1998;5:259-263.

19 Shimizu T, Waga S, Kojima T, Niwa S: Decompression of the vertebral artery for bow-hunter's stroke. Case report. J Neurosurg 1988;69:127-131.

20 Anene-Maidoh TI, Vega RA, Fautheree GL, Reavey-Cantwell JF: An unusual case of pediatric bow hunter's stroke. Surg Neurol Int 2013;4:148.

21 Cornelius JF, George B, N'dri OD, Spiriev T, Steiger HJ, Hänggi D: Bow-hunter's syndrome caused by dynamic vertebral artery stenosis at the cranio-cervical junction - a management algorithm based on a systematic review and a clinical series. Neurosurg Rev 2012;35:127-135; discussion 135.

22 Lu DC, Zador Z, Mummaneni PV, Lawton MT: Rotational vertebral artery occlusion-series of 9 cases. Neurosurgery 2010;67:1066-1072; discussion 1072.

23 Saito K, Hirano M, Taoka T, et al: Juvenile bow hunter's stroke without hemodynamic changes. Clin Med Insights Case Rep 2010;3:1-4.

24 Sakamoto Y, Kimura K, Iguchi Y, et al: An embolic bow hunter's stroke associated with anomaly of cervical spine. Neurology 2011;77:1403-1404.

25 Sakai K, Tsutsui T: Bow hunter's stroke associated with atlantooccipital assimilation - case report. Neurol Med Chir (Tokyo) 1999;39:696-700.

26 Akar Z, Kafadar AM, Tanriover N, et al: Rotational compression of the vertebral artery at the point of dural penetration. Case report. J Neurosurg 2000;93(suppl 2):300-303.

27 Puca A, Scogna A, Rollo M: Craniovertebral junction malformation and rotational occlusion of the vertebral artery. Br J Neurosurg 2000;14:361-364.

28 Robinson BP, Seeger JF, Zak SM: Rheumatoid arthritis and positional vertebrobasilar insufficiency. Case report. J Neurosurg 1986;65:111-114.

29 Chough CK, Cheng BC, Welch WC, Park CK: Bow hunter's stroke caused by a severe facet hypertrophy of C1-2. J Korean Neurosurg Soc 2010;47:134-136.

30 Velat GJ, Reavey-Cantwell JF, Ulm AJ, Lewis SB: Intraoperative dynamic angiography to detect resolution of bow hunter's syndrome: technical case report. Surg Neurol 2006;66:420-423; discussion 423.

31 Vates GE, Wang KC, Bonovich D, Dowd CF, Lawton MT: Bow hunter stroke caused by cervical disc herniation. Case report. J Neurosurg 2002;96(suppl 1):90-93.

32 Kimura T, Sako K, Tohyama Y, Hodozuka A: Bow hunter's stroke caused by simultaneous occlusion of both vertebral arteries. Acta Neurochir (Wien) 1999;141:895-896.

33 Matsuyama T, Morimoto T, Sakaki T: Bow hunter's stroke caused by a nondominant vertebral artery occlusion: case report. Neurosurgery 1997;41:1393-1395.

34 Cloud GC, Markus HS: Diagnosis and management of vertebral artery stenosis. QJM 2003;96:27-54.

35 Husni EA, Storer J: The syndrome of mechanical occlusion of the vertebral artery: further observations. Angiology 1967;18:106-116.

36 Yeh JF, Lin YJ, Po HL, et al: A case of bow hunter's stroke caused by non-dominant vertebral artery. Acta Neurol Taiwan 2005;14:69-73. 
37 Dargon PT, Liang CW, Kohal A, Dogan A, Barnwell SL, Landry GJ: Bilateral mechanical rotational vertebral artery occlusion. J Vasc Surg 2013;58:1076-1079.

38 Saito K, Hirano M, Taoka T, et al: Artery-to-artery embolism with a mobile mural thrombus due to rotational vertebral artery occlusion. J Neuroimaging 2010;20:284-286.

39 Kawasaki T, Dewerchin M, Lijnen HR, Vreys I, Vermylen J, Hoylaerts MF: Mouse carotid artery ligation induces platelet-leukocyte-dependent luminal fibrin, required for neointima development. Circ Res 2001;88:159166.

40 Seki T, Hida K, Akino M, Iwasaki Y: Anterior decompression of the atlantoaxial vertebral artery to treat bow hunter's stroke: technical case report. Neurosurgery 2001;49:1474-1476.

41 Kim K, Isu T, Morimoto D, Kominami S, Kobayashi S, Teramoto A: Anterior vertebral artery decompression with an ultrasonic bone curette to treat bow hunter's syndrome. Acta Neurochir (Wien) 2008;150:301-303; discussion 303.

42 Kamouchi M, Kishikawa K, Matsuo R, et al: Ultrasonographic detection of extracranial vertebral artery compression in bow hunter's brain ischemia caused by neck rotation. Cerebrovasc Dis 2003;16:303-305.

43 Horowitz M, Jovin T, Balzar J, Welch W, Kassam A: Bow hunter's syndrome in the setting of contralateral vertebral artery stenosis: evaluation and treatment options. Spine (Phila Pa 1976) 2002;27:E495-E498.

44 Sugiu K, Agari T, Tokunaga K, Nishida A, Date I: Endovascular treatment for bow hunter's syndrome: case report. Minim Invasive Neurosurg 2009;52:193-195.

45 Iguchi Y, Kimura K, Shibazaki K, Iwanaga T, Ueno Y, Inoue T: Transcranial Doppler and carotid duplex ultrasonography findings in bow hunter's syndrome. J Neuroimaging 2006;16:278-280.

46 Tsutsumi S, Ito M, Yasumoto Y: Simultaneous bilateral vertebral artery occlusion in the lower cervical spine manifesting as bow hunter's syndrome. Neurol Med Chir (Tokyo) 2008;48:90-94.

47 Matsuyama T, Morimoto T, Sakaki T: Comparison of C1-2 posterior fusion and decompression of the vertebral artery in the treatment of bow hunter's stroke. J Neurosurg 1997;86:619-623.

48 Matsuyama T, Morimoto T, Sakaki T: Usefulness of three-dimensional CT for bow hunter stroke. Acta Neurochir (Wien) 1997;139:265-266.

49 Kuether TA, Nesbit GM, Clark WM, Barnwell SL: Rotational vertebral artery occlusion: a mechanism of vertebrobasilar insufficiency. Neurosurgery 1997;41:427-432; discussion 432-433.

50 Choi KD, Choi JH, Kim JS, et al: Rotational vertebral artery occlusion: mechanisms and long-term outcome. Stroke 2013;44:1817-1824.

51 Go G, Hwang SH, Park IS, Park H: Rotational vertebral artery compression: bow hunter's syndrome. J Korean Neurosurg Soc 2013;54:243-245.

52 Andereggen L, Arnold M, Andres RH, Raabe A, Reinert M, Gralla J: Bow hunter's stroke due to prominent degenerative spinal disorder. Clin Neuroradiol 2012;22:355-358.

53 Hanakita J, Miyake H, Nagayasu S, Nishi S, Suzuki T: Angiographic examination and surgical treatment of bow hunter's stroke. Neurosurgery 1988;23:228-232.

54 Miele VJ, France JC, Rosen CL: Subaxial positional vertebral artery occlusion corrected by decompression and fusion. Spine (Phila Pa 1976) 2008;33:E366-E370.

55 Takeshima Y, Nishimura F, Park YS, Nakase H: Fusion surgery for recurrent cerebellar infarctions due to bilateral atlantoaxial rotational vertebral artery occlusion. Spine (Phila Pa 1976) 2014;39:E860-E863. 